\title{
ALGOFLORA AND VASCULAR FLORA OF A LIMESTONE SPRING IN THE WARTA RIVER VALLEY
}

\author{
JOANNA ŻELAZNA-WIECZOREK, MONIKA MAMIŃSKA \\ Department of Algology and Mycology, University of Łódź \\ Banacha 12/16, 90-237 Łódź, Poland \\ e-mail: joannazelaznawieczorek@yahoo.co.uk
}

(Received: July 16, 2005. Accepted: November 16, 2005)

\begin{abstract}
Qualitative analysis of algae, including microhabitats and vascular vegetation in a spring niche, together with basic physical and chemical characteristics is presented. 175 diatom taxa as well as taxa of macroalgae and vascular plants were determined in the spring niche, and the community types were defined. Seasonal variability of diatom communities was observed. The influence of a flood as a catastrophe on the community of diatoms and macroalgae was noticed.
\end{abstract}

KEY WORDS: spring diatoms, macroalgae, vascular plants, and environmental preferences.

\section{INTRODUCTION}

From among as many as 250 springs few were inventoried in the Krakowsko-Wieluńska Upland in 1967 and 1968 (Kleczkowski 1972) and some had been examined algologically. Algae of the region were investigated by Skalska (1966a, b), Skalna $(1969,1973)$ and Kubik (1970). The water gap of the Warta River in the Częstochowska Upland was studied by Waszkiewicz (1999), and the springs of the Pilica river by Kadłubowska (1964).

The examined spring is situated in the old riverbed of the Warta River, in the northern part of the Krakowsko-Wieluńska Upland in Działoszyn, $3 \mathrm{~km}$ east from the border of the Załęczański Landscape Park.

The article presents the results of studies on communities of algae in microhabitats, in association with patches of vascular vegetation colonising them. Additionally the type of communities occurring in the spring is defined. Spring vegetation is an element of central Poland's plant cover, which is poorly explored. Fragmentary findings deal with localities of plant species associated with spring habitats and offer descriptions of the occurring vegetation $(\mathrm{Ku}-$ charski and Filipiak 1999).

Based on qualitative and quantitative diatom analysis, the differentiation within three microhabitats of the springniche communities has been determined. To determine the habitat character, the indicator value for trophic state and pH (Van Dam, Mertens and Sinkeldam 1994) has been attributed to the defined taxa.

\section{STUDY AREA}

The Warta River at the latitude of Działoszyn exemplifies a large natural upland and lowland river, abounding both in islands at different stages of formation and overgrowing, and in old river beds of different age. The landscape is rich in karst springs, concentrated in the Warta valley, as well as defluent springs flowing from sands (Olaczek and Czyżewska 1986).

The examined spring is situated 500 meters south of the Działoszyn-Grady-Łazy road. Geographic co-ordinates of this spring are: longitude $18^{\circ} 54^{\prime}$ east and latitude $51^{\circ} 06^{\prime}$ north. The spring flows out at the foot of the slope (12 $\mathrm{m}$ high; gradient $52.2^{\circ}$ ). Slope aspect is $28.8^{\circ}$ southwest.

The water-bearing layer consists of massy and platy limestone, marls and Rauracin. Spring water flows out close to the Warta old riverbed. As a result of headward erosion, the niche is slightly receded towards the slope. The spring is natural and not used (Dynowska 1983). The bottom is sandy with infrequent rock pieces of limestone origin.

The water flowing from the spring is transparent; the fluvial flow is $6.47 \mathrm{l} / \mathrm{s}$. Water temperature in the spring ranges between 8.6 and $10.3^{\circ} \mathrm{C}$ (Fig. 1); pH: 6.99 and 7.4 (Fig. 2); electrolyte conductivity: 511 and $728 \mu \mathrm{S}$ (Fig. 3). The water level in the examined spring fluctuates between 11.5 and $29 \mathrm{~cm}$, and reached $93 \mathrm{~cm}$ on 24 April 2001 during the flood-stage on the Warta River.

The properties described were different in April 2001 when waters from the Warta River flew into the old river 

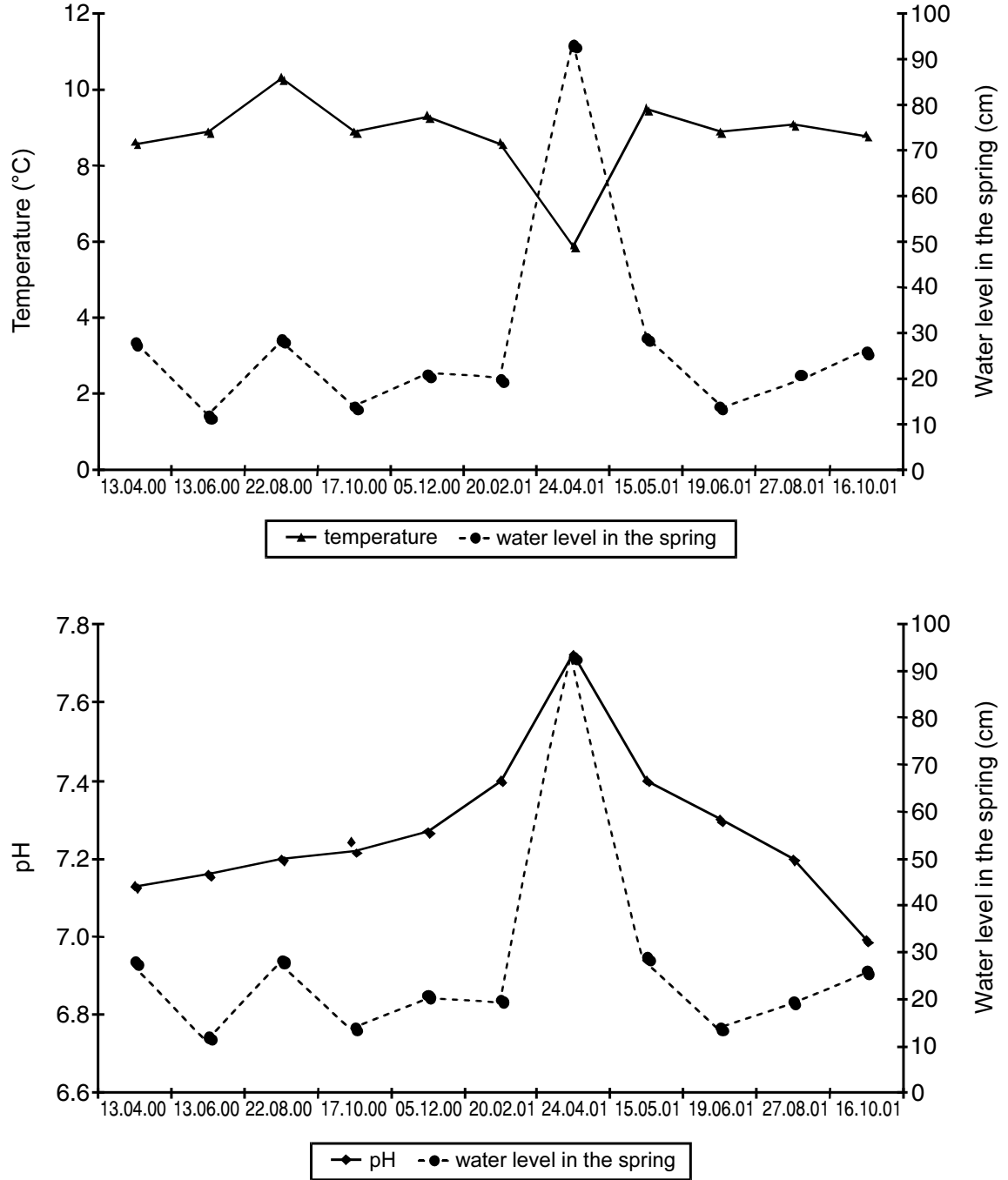

Fig. 1. Temperature and water level in the spring in individual months.

Fig. 2. $\mathrm{pH}$ and water level in the spring in individual months.

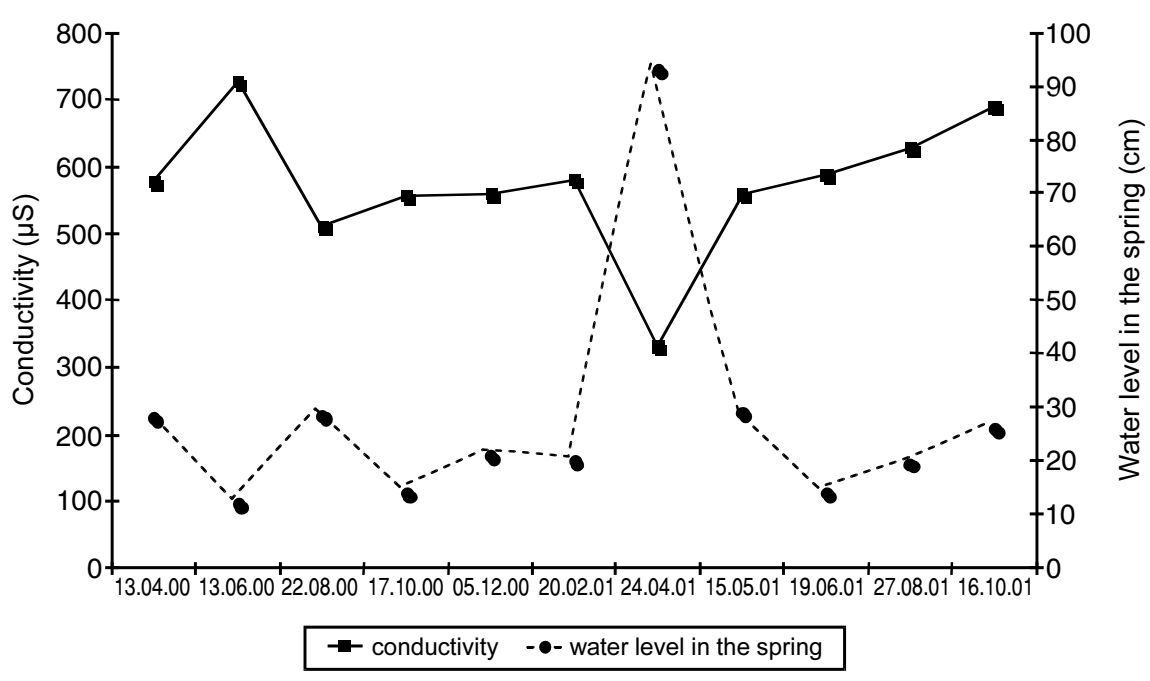

Fig. 3. Conductivity and water level in the spring in individual months. bed. Water temperature was $5.9^{\circ} \mathrm{C}, \mathrm{pH} 7.72$, and conductivity $331 \mu \mathrm{S}$ (Figs $1-3$ ).

\section{METHODS}

Samples for algological studies were collected from a spring situated in the Warta riverbed near Działoszyn. Three sampling sites were established: spring outflow (1.1), one meter downstream from the outflow (1.2), and a stone on the left bank, ca. 0.5 meter from the outflow (1.3).

Sampling in sites 1.1 and 1.2 was carried out every two months: 13 April; 13 June; 22 Aug.; 17 Oct.; 02 Dec. 2000; 20 Feb.; 15 May; 19 June; 27 Aug.; 16 Oct. 2001 (samples were not collected in April 2001 due to the excessively high river stage - flood in the Warta river basin), while in site 1.3, sampling was conducted on 13 June, 22 Aug., 17 Oct. 2000, 19 June, 27 Aug., 16 Oct. 2001. 
TABLE 1. Chemical properties of water collected from the spring.

\begin{tabular}{|c|c|c|}
\hline Day & 17 Sept. 1998 & 18 Oct. 2000 \\
\hline $\mathrm{pH}$ & 7.5 & 7.22 \\
\hline Dissolved oxygen $\mathrm{mg} \mathrm{O}_{2} / \mathrm{dm}^{3}$ & 4.6 & - \\
\hline Nitrates $\mathrm{mg} \mathrm{NO}_{3} / \mathrm{l}$ & 6.0 & 6.63 \\
\hline Organic nitrogen $\mathrm{mg} \mathrm{N}$ org/l & 0.2 & 0.61 \\
\hline Sulfates $\mathrm{mg} \mathrm{SO}_{4} / \mathrm{dm}^{3}$ & 40 & 33 \\
\hline Phosphorus mg P/dm ${ }^{3}$ & 0.06 & 0.08 \\
\hline Sodium $\mathrm{mg} \mathrm{Na} / \mathrm{dm}^{3}$ & 14.2 & - \\
\hline Potassium mg K/dm ${ }^{3}$ & 3.62 & - \\
\hline Calcium mg Ca/dm ${ }^{3}$ & 94 & 100.5 \\
\hline Magnesium mg Mg/dm ${ }^{3}$ & 5.6 & 6.0 \\
\hline Chlorine $\mathrm{mg} \mathrm{Cl} / \mathrm{dm}^{3}$ & - & 27.2 \\
\hline Total hardness $\mathrm{mg} \mathrm{CaCO}_{3} / \mathrm{dm}^{3}$ & 260 & 275 \\
\hline Alkalinity to methyl orange $\mathrm{mgCaCO} / \mathrm{dm}^{3}$ & - & 221 \\
\hline
\end{tabular}

The benthos was collected with a pipette from the surface layer of the substrate, at the outflow, one meter from the outflow and from the stone. Occurring filamentous algae were also collected. Samples were kept in a refrigerator for no longer than three days for purposes of observation and classification of live material. Temperature, water $\mathrm{pH}$, electrolyte conductivity, water level in the spring, and water level in the Warta were measured at time of sampling.

The specification of chemical properties in the spring is based on the water analysis performed in the laboratory of the Province Inspectorate of Environmental Protection in Łódź on 17 Sept. 1998 and 18 Oct. 2000 (Table 1).

The spring niche was charted in the scale of 1:20 on each event (Fig. 4), and the vegetation distribution was presented on map.

Macroalgae were observed and determined live, using microscope magnification $12.5 \times 10$ and $12.5 \times 40$. All slides were viewed whole in parallel lines from left to right. The samples were then fixed in $40 \%$ formalin. In order to obtain clean diatom frustules, the collected benthic material was digested. To make permanent slides, the diatom material was mounted in synthetic resin Naphrax (NBS).

Microscopic slides were examined by MB-30 microscope, using magnification $12.5 \times 10,12.5 \times 40$ and $12.5 \times 100$. The slides were viewed and examined using three lines located at $1 / 4,1 / 2$ and $3 / 4$ of the width of the cover glass $20 \times 20 \mathrm{~mm}$.

Diatoms were classified following Krammer and LangeBertalot (1986, 1988, 1991a, b), Lange-Bertalot (2001), Lange-Bertalot and Metzeltin (1996), macroalge by Star-

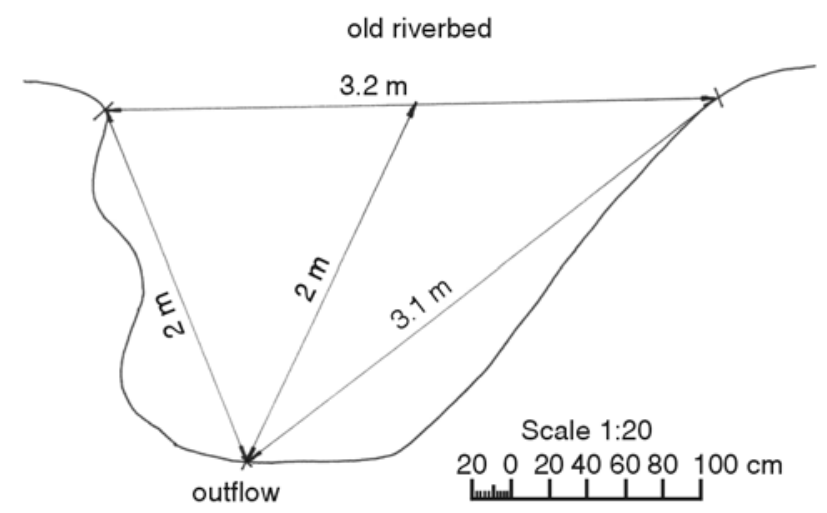

Fig. 4. Spring niche parameters. mach (1968, 1972, 1977), whereas vascular plants using Szafer et al. (1986).

The following issues were considered in the analysis of diatom communities on the basis of permanent slides:

- quantitative differentiation expressed in percentage participation of individual species;

- occurrence constancy according to the V-grade BraunBlanquet scale: $81-100 \%$ (V class), 61-80\% (IV class), $41-$ $60 \%$ (III class), $21-40 \%$ (II class), 1-20\% (I class); for site 1.1 and site 1.2 based on 10 samples, for site 1.3 based on 6 samples;

- dominant species with the participation above 5\% individuals, subdominant species $2-5 \%$, influents $1-2 \%$, accessory and alien species below 1\% (Trojan 1975);

- the Kulczyński similarity index was used to determine degree of similarity of the communities in individual months (Kawecka and Eloranta 1994):

$$
\mathrm{K}=(\mathrm{c} / 2)(1 / \mathrm{a}+1 / \mathrm{b})
$$

$\mathrm{a}$ - number of species in community $\mathrm{A} ; \mathrm{b}$ - number of species in community $\mathrm{B}$; $\mathrm{c}-$ number of species common for both communities.

To the identified diatom taxa, indicatory values determining their tolerance to trophic state (TS), $\mathrm{pH}$, based on Van Dam et al. (1994) checklist were attributed:

- TS 1 - oligotraphentic, 2 - oligo-mesotraphentic, 3 mesotraphentic, 4 - meso-eutraphentic, 5 - eutraphentic, 6 - hypereutraphentic, 7 - oligo- to eutraphentic (hypereutraphentic);

- pH 1 - acidobiontic, 2 - acidophilus, 3 - circumneutral, 4 - alkaliphilous, 5 - alkalibiontic, 6 - indifferent;

0 - marked taxa with undetermined indicator value.

\section{RESULTS}

The studied spring is characterized by low, stabile annual water temperature, their high conductivity; water effluents from marvel rocks are medium hard with high calcium concentration, with appearing gain of organic nitrogen during the period of the study.

In 26 samples 208 taxa of diatoms were determined. In site 1.1., 174 taxa of diatoms were determined. The taxa that occur exclusively in this place were: Achnanthes ploenensis, Campylodiscus hibernicus, Cyclotella stelligera, Cymbella aspera, C. cistula, Gomphonema tenue, Hippodonta hungarica, Navicula rostellata, Nitzschia pellucida (Table 2). In site 1.2., 191 taxa were determined. The taxa that occur exclusively in site 1.2 were: Achnanthes nodosa, Caloneis leptosoma, Cymbella amphicephala, C. microcephala, C. proxima, Diatoma mesodon, Diploneis ovalis, Eunotia soleirolii, Gomphonema productum, Luticola nivalis, Nitzschia sigmoidea, N. sinuata, Pinnularia gibba, $P$. legumen, Surirella biseriata, S. linearis (Table 2). In site 1.3., 172 taxa were identified. The taxa found exclusively in this place were: Achnanthes rechtensis, Mayamea excelsa, Navicula capitatoradiata, N. veneta (Table 2). For these three sampling sites 150 taxa were common.

In site 1.1, the number of taxa gradually decreased from 89 in April 2000 to 65 in Dec. 2000. The number of taxa increased between Feb. 2001 and June 2001, when it reached its maximum level of 110 (Fig. 5). In site 1.2, the 
TABLE 2. List of diatom taxa identified in this spring niche.

A - constancy class for species in site 1.1; B - constancy class for species in site 1.2; C - constancy class for species in site 1.3 .

TS - trophic state (Van Dam et al. 1994); pH (Van Dam et al. 1994); 0 - unspecified

\begin{tabular}{|c|c|c|c|c|c|}
\hline Taxa & A & B & $\mathrm{C}$ & TS & $\mathrm{pH}$ \\
\hline Achnantheiopsis delicatula (Kutzing) Lange-Bertalot & I & I & II & 0 & 5 \\
\hline Achnantheiopsis dubia (Grunow) Lange-Bertalot & $\mathrm{V}$ & V & $\mathrm{V}$ & 0 & 0 \\
\hline Achnanthes biasolettiana var. biasolettiana Grunow & III & III & IV & 0 & 0 \\
\hline Achnanthes biasolettiana var. subatomus Lange-Bertalot & I & I & II & 3 & 4 \\
\hline Achnanthes bioretti Germain & IV & $\mathrm{V}$ & $\mathrm{V}$ & 3 & 3 \\
\hline Achnanthes chlidanos Hohn \& Hellerman & I & & I & 0 & 0 \\
\hline Achnanthes clevei var. bottnica Cleve & III & IV & IV & 0 & 0 \\
\hline Achnanthes conspicua Mayer & IV & IV & III & 7 & 3 \\
\hline Achnanthes hungarica (Grunow) Grunow & III & III & III & 6 & 4 \\
\hline Achnanthes lauenburgiana Hustedt & II & I & I & 2 & 3 \\
\hline Achnanthes nodosa Cleve & & I & & 0 & 0 \\
\hline Achnanthes ploenensis Hustedt & I & & & 4 & 4 \\
\hline Achnanthes rechtensis Leclerq & & & I & 1 & 3 \\
\hline Achnanthes rossii Hustedt & I & II & I & 1 & 3 \\
\hline Achnanthidium minutissimum (Kutzing) Czarnecki & $\mathrm{V}$ & $\mathrm{V}$ & V & 7 & 3 \\
\hline Achnanthidium minutissimum var. affinis & $\mathrm{V}$ & IV & IV & 0 & 4 \\
\hline Adlafia minuscula (Grunow) Lange-Bertalot & III & III & III & 1 & 4 \\
\hline Amphora fogediana Krammer & III & IV & III & 0 & 0 \\
\hline Amphora inariensis Krammer & $\mathrm{V}$ & $\mathrm{V}$ & $\mathrm{V}$ & 1 & 0 \\
\hline Amphora libyca Ehrenberg & V & $\mathrm{V}$ & V & 0 & 0 \\
\hline Amphora ovalis (Kützing) Kützing & III & $\mathrm{V}$ & V & 5 & 4 \\
\hline Amphora pediculus (Kützing) Grunow & $\mathrm{V}$ & $\mathrm{V}$ & $\mathrm{V}$ & 5 & 4 \\
\hline Anomoeoneis sphaerophora (Ehrenberg) Pfitzer & I & II & & 5 & 5 \\
\hline Aulacoseira granulata (Ehrenberg) Simonsen & II & I & I & 5 & 4 \\
\hline Caloneis amphisbaena (Bory) Cleve & II & II & IV & 5 & 4 \\
\hline Caloneis bacillum (Grunow) Cleve & III & III & III & 4 & 4 \\
\hline Caloneis leptosoma (Grunow) Krammer & & I & & 2 & 3 \\
\hline Caloneis silicula (Ehrenberg) Cleve & I & III & III & 4 & 4 \\
\hline Campylodiscus hybernicus Ehrenberg & I & & & 5 & 5 \\
\hline Cavinula lapidosa (Krasske) Lange-Bertalot & & I & II & 0 & 2 \\
\hline Cocconeis neodiminuta Krammer & IV & IV & IV & 0 & 0 \\
\hline Cocconeis placentula var. euglypta (Ehrenberg) Grunow & IV & $\mathrm{V}$ & $\mathrm{V}$ & 5 & 4 \\
\hline Cocconeis placentula var. lineata (Ehrenberg) Van Heurck & III & IV & II & 5 & 4 \\
\hline Cocconeis placentula var. placentula Ehrenberg & $\mathrm{V}$ & $\mathrm{V}$ & $\mathrm{V}$ & 5 & 4 \\
\hline Cocconeis placentula var. pseudolineata Geitler & II & III & III & 0 & 0 \\
\hline Craticula cuspidata (Kützing) D.G. Mann & I & I & I & 5 & 4 \\
\hline Cyclotella meneghiniana Kützing & III & III & IV & 5 & 4 \\
\hline Cyclotella radiosa (Grunow) Lemmermann & III & III & II & 5 & 4 \\
\hline Cyclotella stelligera Cleve \& Grunow & I & & & 0 & 0 \\
\hline Cymatopleura solea var. apiculata (Smith) Ralfs & II & $\mathrm{I}$ & & 0 & 0 \\
\hline Cymatopleura solea var. solea (Brebisson) Smith & III & III & II & 5 & 4 \\
\hline Cymbella affinis Kützing & & I & I & 5 & 4 \\
\hline Cymbella amphicecephala Naegelt & & $\mathrm{I}$ & & 2 & 3 \\
\hline Cymbella aspera (Ehrenberg) Peragello & I & & & 7 & 4 \\
\hline Cymbella cistula (Ehrenberg) Kirchner & I & & & 5 & 4 \\
\hline Cymbella cuspidata Kützing & I & I & & 0 & 3 \\
\hline Cymbella microcephala Grunow & & I & & 4 & 4 \\
\hline Cymbella naviculiformis (Auerswald) Cleve & III & IV & III & 5 & 3 \\
\hline Cymbella proxima Reimer & & I & & 3 & 0 \\
\hline Diadesmis contenta (Grunow) D.G. Mann & III & I & II & 7 & 4 \\
\hline Diadesmis perpusilla (Grunow) D.G. Mann & I & & I & 1 & 3 \\
\hline Diatoma mesodon (Ehrenberg) Kützing & & $\mathrm{I}$ & & 3 & 3 \\
\hline Diatoma tenuis Agardh & I & III & I & 5 & 4 \\
\hline Diatoma vulgaris Bory & II & II & II & 4 & 5 \\
\hline Diploneis oblongella (Naegeli) Cleve-Euler & III & III & V & 0 & 4 \\
\hline Diploneis oculata (Brebisson) Cleve & I & II & I & 0 & 3 \\
\hline Diploneis ovalis (Hilse) Cleve & & I & & 0 & 4 \\
\hline Encyonema minutum (Hilse) Mann & & I & I & 0 & 3 \\
\hline Encyonema silesiacum (Bleisch) Mann & IV & IV & IV & 7 & 3 \\
\hline Epithemia adnata (Kützing) Brebisson & III & II & I & 4 & 5 \\
\hline Eunotia bilunaris var. bilunaris (Ehrenberg) Mills & $\mathrm{V}$ & $\mathrm{V}$ & $\mathrm{V}$ & 7 & 6 \\
\hline Eunotia bilunaris var. mucophila Lange-Bertalot \& Nörpel & II & II & & 2 & 2 \\
\hline Eunotia incisa var. incisa Gregory & I & $\mathrm{I}$ & I & 1 & 2 \\
\hline Eunotia minor (Kützing) Grunow & IV & II & III & 0 & 2 \\
\hline Eunotia soleiriolii (Kützing) Rabenhorst & & II & & 1 & 3 \\
\hline
\end{tabular}


TABLE 2. Cont.

\begin{tabular}{|c|c|c|c|c|c|}
\hline Taxa & A & $\mathrm{B}$ & $\mathrm{C}$ & TS & $\mathrm{pH}$ \\
\hline Fallacia insociabilis (Krasske) D.G. Mann & & $\mathrm{I}$ & II & 3 & 3 \\
\hline Fallacia lenzii (Hustedt) Lange-Bertalot & II & III & II & 0 & 4 \\
\hline Fragilaria biceps (Kützing) Lange-Bertalot & & I & I & 5 & 4 \\
\hline Fragilaria brevistriata Grunow & I & III & IV & 7 & 4 \\
\hline Fragilaria capucina var. vaucheriae (Kützing) Lange-Bertalot & II & II & II & 5 & 4 \\
\hline Fragilaria capucina var. capucina Desmazieres & II & II & I & 3 & 3 \\
\hline Fragilaria capucina var. gracilis (Oestrup) Hustedt & III & IV & $\mathrm{I}$ & 2 & 3 \\
\hline Fragilaria capucina var. mesolepta (Rabenhorst) Rabenhorst & II & II & III & 0 & 0 \\
\hline Fragilaria capucina var. rumpens (Kützing) Lange-Bertalot & I & I & I & 2 & 3 \\
\hline Fragilaria construens $\mathrm{f}$. binodis (Ehrenberg) Hustedt & III & III & V & 4 & 4 \\
\hline Fragilaria construens f. construens (Ehrenberg) Grunow & III & III & III & 4 & 4 \\
\hline Fragilaria construens $\mathrm{f}$. venter (Ehrenberg) Hustedt & IV & IV & $\mathrm{V}$ & 4 & 4 \\
\hline Fragilaria delicatissima (Brebisson) Lange-Bertalot & I & & I & 3 & 3 \\
\hline Fragilaria dilatata (Brebisson) Lange-Bertalot & & I & I & 5 & 4 \\
\hline Fragilaria leptostauron var. leptostauron (Ehrenberg) Hustedt & $\mathrm{V}$ & $\mathrm{V}$ & V & 4 & 4 \\
\hline Fragilaria nitzschioides Grunow & & $\mathrm{I}$ & I & 0 & 0 \\
\hline Fragilaria parasitica var. parasitica (Smith) Grunow & II & III & IV & 4 & 4 \\
\hline Fragilaria parasitica var. subconstricta Grunow & II & IV & III & 4 & 4 \\
\hline Fragilaria pinnata var. intercedens (Grunow) Hustedt & IV & IV & V & 0 & 0 \\
\hline Fragilaria pinnata var. pinnata Ehrenberg & V & $\mathrm{V}$ & V & 7 & 4 \\
\hline Fragilaria ulna var. acus (Kützing) Lange-Bertalot & III & I & IV & 5 & 4 \\
\hline Fragilaria ulna var. ulna (Nitzsch) Lange-Bertalot & V & $\mathrm{V}$ & V & 7 & 4 \\
\hline Frustulia vulgaris (Thwaites) De Toni & $\mathrm{V}$ & $\mathrm{V}$ & IV & 4 & 4 \\
\hline Geissleria decussis (Ostrup) Lange-Bertalot et Metzeltin s.l. & III & $\mathrm{V}$ & $\mathrm{V}$ & 4 & 4 \\
\hline Gomphonema acuminatum Ehrenberg & III & IV & IV & 5 & 4 \\
\hline Gomphonema angustum Agardh & II & I & II & 1 & 4 \\
\hline Gomphonema clavatum Ehrenberg & III & III & I & 4 & 3 \\
\hline Gomphonema gracile Ehrenberg & I & I & & 3 & 3 \\
\hline Gomphonema insigne Gregory & I & I & I & 0 & 0 \\
\hline Gomphonema micropus Kützing & IV & $\mathrm{V}$ & V & 5 & 4 \\
\hline Gomphonema minutum (Agardh) Agardh & II & I & I & 5 & 3 \\
\hline Gomphonema olivaceum (Hornemann) Brebisson & III & III & IV & 5 & 5 \\
\hline Gomphonema parvulum (Kützing) Kützing & IV & $\mathrm{V}$ & $\mathrm{V}$ & 5 & 3 \\
\hline Gomphonema productum (Grunow) Lange-Bertalot \& Raichardt & & I & & 2 & 3 \\
\hline Gomphonema sarcophagus Gregory & IV & IV & V & 3 & 4 \\
\hline Gomphonema tenue Fricke & I & & & 0 & 0 \\
\hline Gomphonema truncatum Ehrenberg & IV & $\mathrm{V}$ & I & 4 & 4 \\
\hline Gyrosigma acuminatum (Kützing) Rabenhorst & $\mathrm{V}$ & $\mathrm{V}$ & V & 5 & 5 \\
\hline Hantzschia amphioxys (Ehrenberg) Grunow & I & III & IV & 7 & 3 \\
\hline Hippodonta capitata (Ehrenberg) Lange-Bertalot, Metzeltin et Witkowski & $\mathrm{V}$ & $\mathrm{V}$ & V & 4 & 4 \\
\hline Hippodonta costulata (Grunow) Lange-Bertalot, Metzeltin et Witkowski & IV & $\mathrm{V}$ & $\mathrm{V}$ & 0 & 4 \\
\hline Hippodonta hungarica (Grunow) Lange-Bertalot, Metzeltin et Witkowski & I & & & 4 & 4 \\
\hline Luticola mutica (Kützing) D. G. Mann & & II & I & 5 & 3 \\
\hline Luticola nivalis (Ehrenberg) D.G. Mann & & I & & 5 & 3 \\
\hline Mayamaea fossalis (Krasske) Lange-Bertalot var. fossalis & I & I & I & 0 & 3 \\
\hline Mayamea asellus (Weinhold) Lange-Bertalot & & I & I & 0 & 4 \\
\hline Mayamea atomus (Kützing) Lange-Bertalot & $\mathrm{I}$ & $\mathrm{I}$ & & 6 & 4 \\
\hline Mayamea excelsa (Krasske) Lange-Bertalot & & & I & 6 & 3 \\
\hline Melosira varians Agardh & $\mathrm{V}$ & $\mathrm{V}$ & V & 5 & 4 \\
\hline Meridion circulare (Greville) Agardh & V & $\mathrm{V}$ & V & 7 & 4 \\
\hline Navicula capitatoradiata Germain & & & I & 5 & 4 \\
\hline Navicula cari Ehrenberg & & $\mathrm{I}$ & II & 7 & 0 \\
\hline Navicula cincta (Ehrenberg) Ralf & $\mathrm{I}$ & II & IV & 5 & 4 \\
\hline Navicula cryptocephala Kützing & IV & $\mathrm{V}$ & $\mathrm{V}$ & 7 & 3 \\
\hline Navicula cryptotenella Lange-Bertalot & IV & IV & V & 7 & 4 \\
\hline Navicula exilis Kützing & V & $\mathrm{V}$ & $\mathrm{V}$ & 0 & 0 \\
\hline Navicula graciloides Mayer & II & IV & IV & 5 & 5 \\
\hline Navicula gregaria Donkin & V & $\mathrm{V}$ & V & 5 & 4 \\
\hline Navicula integra (Smith) Ralfs & II & $\mathrm{V}$ & III & 5 & 3 \\
\hline Navicula joubaudii Germain & $\mathrm{V}$ & $\mathrm{V}$ & $\mathrm{V}$ & 0 & 0 \\
\hline Navicula lanceolata (Agardh) Ehrenberg & III & III & III & 5 & 4 \\
\hline Navicula menisculus Schumann & IV & IV & IV & 5 & 4 \\
\hline Navicula minima Grunow & V & $\mathrm{V}$ & V & 5 & 4 \\
\hline Navicula moskali Witkowski \& Lange-Bertalot & $\mathrm{V}$ & $\mathrm{V}$ & V & 0 & 0 \\
\hline Navicula oblonga (Kützing) Kützing & II & IV & II & 5 & 4 \\
\hline Navicula oppungata Hustedt & & II & I & 0 & 0 \\
\hline Navicula protracta (Grunow) Cleve & IV & IV & V & 5 & 3 \\
\hline
\end{tabular}


TABLE 2. Cont.

\begin{tabular}{|c|c|c|c|c|c|}
\hline Taxa & A & $\mathrm{B}$ & $\mathrm{C}$ & TS & $\mathrm{pH}$ \\
\hline Navicula pygmaea Kützing & II & II & I & 5 & 5 \\
\hline Navicula radiosa Kützing & III & $\mathrm{V}$ & II & 4 & 3 \\
\hline Navicula reinhardtii (Grunow) Grunow \& Möller & IV & $\mathrm{V}$ & $\mathrm{V}$ & 5 & 5 \\
\hline Navicula rhynchocephala Kützing & $\mathrm{I}$ & II & I & 7 & 4 \\
\hline Navicula rostellata (Kützing) Lange-Bertalot & I & & & 5 & 4 \\
\hline Navicula seminulum Grunow & II & I & IV & 5 & 3 \\
\hline Navicula slesvicensis Grunow & $\mathrm{I}$ & II & & 5 & 4 \\
\hline Navicula tenelloides Hustedt & III & IV & $\mathrm{V}$ & 5 & 4 \\
\hline Navicula tripunctata (O.F. Müller) Bory & III & $\mathrm{I}$ & II & 5 & 4 \\
\hline Navicula trivialis Lange-Bertalot & $\mathrm{I}$ & II & & 5 & 4 \\
\hline Navicula upsaliensis Grunow & $\mathrm{V}$ & $\mathrm{V}$ & $\mathrm{V}$ & 0 & 4 \\
\hline Navicula veneta Kützing & & & I & 5 & 4 \\
\hline Neidium affine (Ehrenberg) Pfitzer & III & II & II & 4 & 3 \\
\hline Neidium ampliatum (Ehrenberg) Krammer & II & IV & III & 2 & 3 \\
\hline Neidium binodeforme Krammer & IV & IV & IV & 0 & 0 \\
\hline Neidium bisulcatum (Lagerstedt) Cleve & I & IV & & 1 & 3 \\
\hline Neidium dubium (Ehrenberg) Cleve & $\mathrm{V}$ & $\mathrm{V}$ & $\mathrm{V}$ & 4 & 3 \\
\hline Nitzschia acicularis (Kützing) Smith & II & II & & 5 & 4 \\
\hline Nitzschia acidoclinata Lange-Bertalot & $\mathrm{I}$ & II & II & 3 & 3 \\
\hline Nitzschia agnita Hustedt & II & II & & 0 & 0 \\
\hline Nitzschia amphibia Grunow & IV & $\mathrm{V}$ & $\mathrm{V}$ & 5 & 4 \\
\hline Nitzschia capitellata Hustedt & IV & $\mathrm{V}$ & $\mathrm{V}$ & 6 & 4 \\
\hline Nitzschia constricta (Kützing) Ralfs & $\mathrm{V}$ & $\mathrm{V}$ & $\mathrm{V}$ & 5 & 4 \\
\hline Nitzschia debilis (Arnott) Grunow \& Grunow & III & II & II & 0 & 4 \\
\hline Nitzschia dissipata var. dissipata (Kützing) Grunow & IV & $\mathrm{V}$ & $\mathrm{V}$ & 4 & 4 \\
\hline Nitzschia dissipata var. media (Hantzsch) Grunow & IV & III & II & 0 & 4 \\
\hline Nitzschia dubia Smith & $\mathrm{V}$ & IV & I & 5 & 3 \\
\hline Nitzschia fonticola Grunow & IV & $\mathrm{V}$ & V & 4 & 4 \\
\hline Nitzschia fossilis (Grunow) Grunow & & I & I & 0 & 2 \\
\hline Nitzschia frustulum (Kützing) Grunow & III & III & II & 5 & 4 \\
\hline Nitzschia gracilis Hantzsch & III & II & II & 3 & 3 \\
\hline Nitzschia hungarica Grunow & III & III & II & 5 & 4 \\
\hline Nitzschia inconspicua Grunow & $\mathrm{I}$ & II & III & 5 & 4 \\
\hline Nitzschia levidensis var. levidensis (Smith) Grunow & I & II & I & 5 & 4 \\
\hline Nitzschia linearis var. linearis (Agardh) Smith & $\mathrm{V}$ & $\mathrm{V}$ & V & 4 & 4 \\
\hline Nitzschia linearis var. subtilis (Grunow) Hustedt & II & II & I & 0 & 0 \\
\hline Nitzschia palea (Kützing) Smith & IV & $\mathrm{V}$ & $\mathrm{V}$ & 6 & 3 \\
\hline Nitzschia paleacea (Grunow) Grunow & IV & IV & IV & 5 & 4 \\
\hline Nitzschia pellucida Grunow & I & & & 0 & 0 \\
\hline Nitzschia pusilla Grunow & $\mathrm{V}$ & III & V & 7 & 3 \\
\hline Nitzschia recta Hantzsch & II & III & I & 7 & 4 \\
\hline Nitzschia sigmoidea (Nitzsch) Smith & & I & & 5 & 4 \\
\hline Nitzschia sinuata (Thwaites) Grunow & & I & & 5 & 4 \\
\hline Nitzschia sociabilis Hustedt & $\mathrm{I}$ & & II & 5 & 3 \\
\hline Nitzschia tubicola Grunow & $\mathrm{I}$ & II & I & 0 & 0 \\
\hline Paconeis elginensis (Gregory) Cox & $\mathrm{I}$ & III & III & 5 & 4 \\
\hline Pinnularia appendiculata (Agardh) Cleve & & I & II & 2 & 2 \\
\hline Pinnularia borealis var. rectangularis Carison & II & II & II & 0 & 0 \\
\hline Pinnularia gibba Ehrenberg & & I & & 7 & 3 \\
\hline Pinnularia legumen (Ehrenberg) Ehrenberg & & I & & 1 & 3 \\
\hline Pinnularia lundi var. linearis Hustedt & III & IV & II & 3 & 2 \\
\hline Pinnularia maior (Kützing) Rabenhorst & I & I & I & 4 & 3 \\
\hline Pinnularia microstauron (Ehrenberg) Cleve & & II & II & 7 & 3 \\
\hline Pinnularia nodosa (Ehrenberg) Smith & II & I & I & 1 & 2 \\
\hline Pinnularia viridis (Nitzsch) Ehrenberg & III & III & II & 7 & 3 \\
\hline Placoneis clementis (Grunow) Cox & $\mathrm{I}$ & II & II & 4 & 4 \\
\hline Placoneis gastrum (Ehrenberg) Mereschkowsky & I & II & III & 5 & 4 \\
\hline Placoneis placentula (Ehrenberg) Cox & II & III & IV & 5 & 4 \\
\hline Placoneis subplacentula Hustedt & III & IV & $\mathrm{V}$ & 0 & 0 \\
\hline Planothidium frequentissimum (Lange-Bertalot) Lange-Bertalot & $\mathrm{V}$ & $\mathrm{V}$ & $\mathrm{V}$ & 7 & 4 \\
\hline Planothidium lanceolatum (Brebisson) Lange-Bertalot & $\mathrm{V}$ & $\mathrm{V}$ & $\mathrm{V}$ & 5 & 4 \\
\hline Rhoicosphaenia abbreviata (Agardh) Lange-Bertalot & $\mathrm{V}$ & $\mathrm{V}$ & $\mathrm{V}$ & 5 & 4 \\
\hline Sellaphora bacillum (Ehrenberg) Mann & III & IV & IV & 4 & 4 \\
\hline Sellaphora laevissima (Kützing) Lange-Bertalot & III & IV & IV & 3 & 3 \\
\hline Sellaphora pupula (Kutzing) Mereschkowsky s.l. & $\mathrm{V}$ & $\mathrm{V}$ & $\mathrm{V}$ & 4 & 3 \\
\hline Stauroneis anceps Ehrenberg & II & $\mathrm{V}$ & $\mathrm{V}$ & 4 & 3 \\
\hline Stauroneis kriegerii Patrick & $\mathrm{V}$ & $\mathrm{V}$ & $\mathrm{V}$ & 4 & 3 \\
\hline
\end{tabular}




\begin{tabular}{|c|c|c|c|c|c|}
\hline Taxa & A & $\mathrm{B}$ & $\mathrm{C}$ & TS & $\mathrm{pH}$ \\
\hline Stauroneis lauenburgiana Hustedt & I & $\mathrm{I}$ & I & 0 & 0 \\
\hline Stauroneis legumen (Ehrenberg) Kützing & II & II & I & 4 & 3 \\
\hline Stauroneis phoenicentron (Nitzsch) Ehrenberg & III & IV & II & 4 & 3 \\
\hline Stauroneis smithii Grunow & V & $\mathrm{V}$ & V & 7 & 4 \\
\hline Stephanodiscus hantzschii Grunow & V & IV & IV & 6 & 5 \\
\hline Surirella angusta Kützing & IV & $\mathrm{V}$ & IV & 5 & 4 \\
\hline Surirella biseriata Brebisson & & I & & 5 & 4 \\
\hline Surirella briebissoni var. kuetzingii Krammer \& Lange-Bertalot & II & III & III & 5 & 4 \\
\hline Surirella linearis Smith & & II & & 2 & 3 \\
\hline
\end{tabular}

number of taxa also decreased from its maximum number of 126 in April 2000, to reach the lowest number of 73 in Feb. 2001. Their number started to increase in May 2001 to reach the number of 122 in Oct. 2001 (Fig. 5). In site 1.3, the number of taxa was constant in June and Oct. 2000, and equalled 89. A slight increase to 93 taxa was recorded in August. In 2001, the number of taxa increased from 94 in June to the maximum number of 121 in Aug., and decreased to 100 in October (Fig. 5).

The following groups of diatom taxa were differentiated: dominants, subdominants, influents, accessory and rare species. Dominants were differentiated and are presented in Figure 6, in order to present the taxa that characterise a given diatom community the best. Dominant species were: Achnantheiopsis dubia (with max. participation $70.1 \%)$, Melosira varians (60.9\%), Nitzschia linearis var. linearis (46.5\%), Navicula joubaudii (45.23\%), Planothidium lanceolatum (15.95\%), Hippodonta capitata (15.79\%), Fragilaria ulna var. ulna (12.15\%), Achnanthes biasolettiana var. biasolettiana (9.54\%), Nitzschia capitellata $(9.54 \%)$, Fragilaria pinnata $(8.78 \%)$, Nitzschia fonticola (7.73\%), Achnanthidium minutissimum (7.13\%), Stephanodiscus hantzschii (7.05\%), Amphora pediculus (6.3\%), Meridion circulare $(5.43 \%)$, Cocconeis placentula var. placentula $(5.33 \%)$.

For the diatom taxa determined the constancy grades were ascribed according to the Braun-Blanquet method, commonly used in phytosociology. For sites 1.1, 1.2 and 1.3, the taxa occurring in individual classes were grouped together and are presented in Table 3.

In site 1.1, the number of specimens fluctuations was recorded, reaching the highest value in June 2001-4412. The lowest number of specimens values was recorded in Oct.
TABLE 3. Number of taxa in sites 1.1, 1.2 and 1.3 with ascribed constancy classes.

\begin{tabular}{lccccc}
\hline $\begin{array}{c}\text { Number } \\
\text { of taxa } \\
\text { in sites }\end{array}$ & V & IV & III & II & I \\
\cline { 2 - 6 } 1.1 & 34 & 26 & 38 & 32 & 45 \\
1.2 & 49 & 31 & 29 & 35 & 47 \\
1.3 & 54 & 25 & 31 & 20 & 42 \\
\hline
\end{tabular}

2001-375 (Fig. 7). In site 1.2, a high number of specimens was recorded in Oct. 2000-7385. Whereas the lowest one in May 2001-602 (Fig. 7). In site 1.3, the number of specimens did not fluctuate greatly in the months of June, August and October 2000, and ranged between 2865 and 3059. In June 2001, a frequency increase was observed and as many as 4469 specimens were recorded. Afterwards, the number of specimens began to fall and reached its lowest value of 827 in October (Fig. 7).

Diatom communities, representing three microhabitats, were compared on the basis of the qualitative comparison index, called the Kulczyński similarity index (Kawecka and Eloranta 1994). The greatest value of the similarity coefficient 0.82 , was recorded in June 2001 between the outflow and one meter from the outflow, while the smallest one 0.65 in Feb. 2001, between the same sites. In other cases, the similarity coefficient was constant (0.71-0.78).

To the identified diatom taxa, indicator values for $\mathrm{pH}$ and trophic state (Table 2) have been attributed. According to trophic state, most taxa belong to meso- and eutraphentic, however in respect of - to alkalophilic (Fig. 8). The trophic state for 45 identified taxa as well as $\mathrm{pH}$ value for 31 taxa, have not been determined, so far.

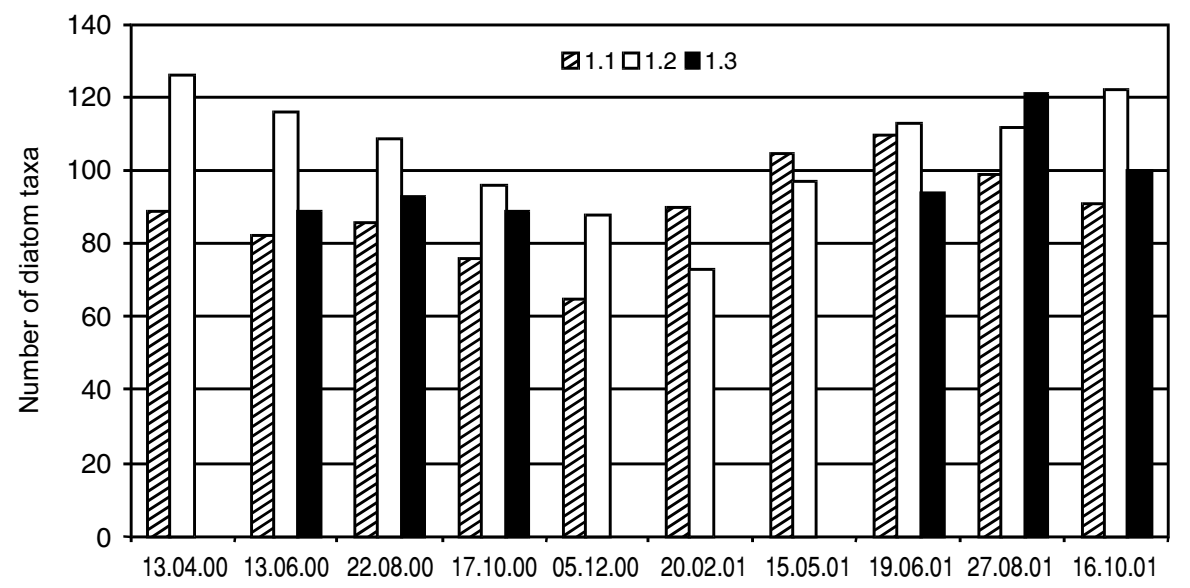

Fig. 5. Number of diatom taxa in individual samples. 

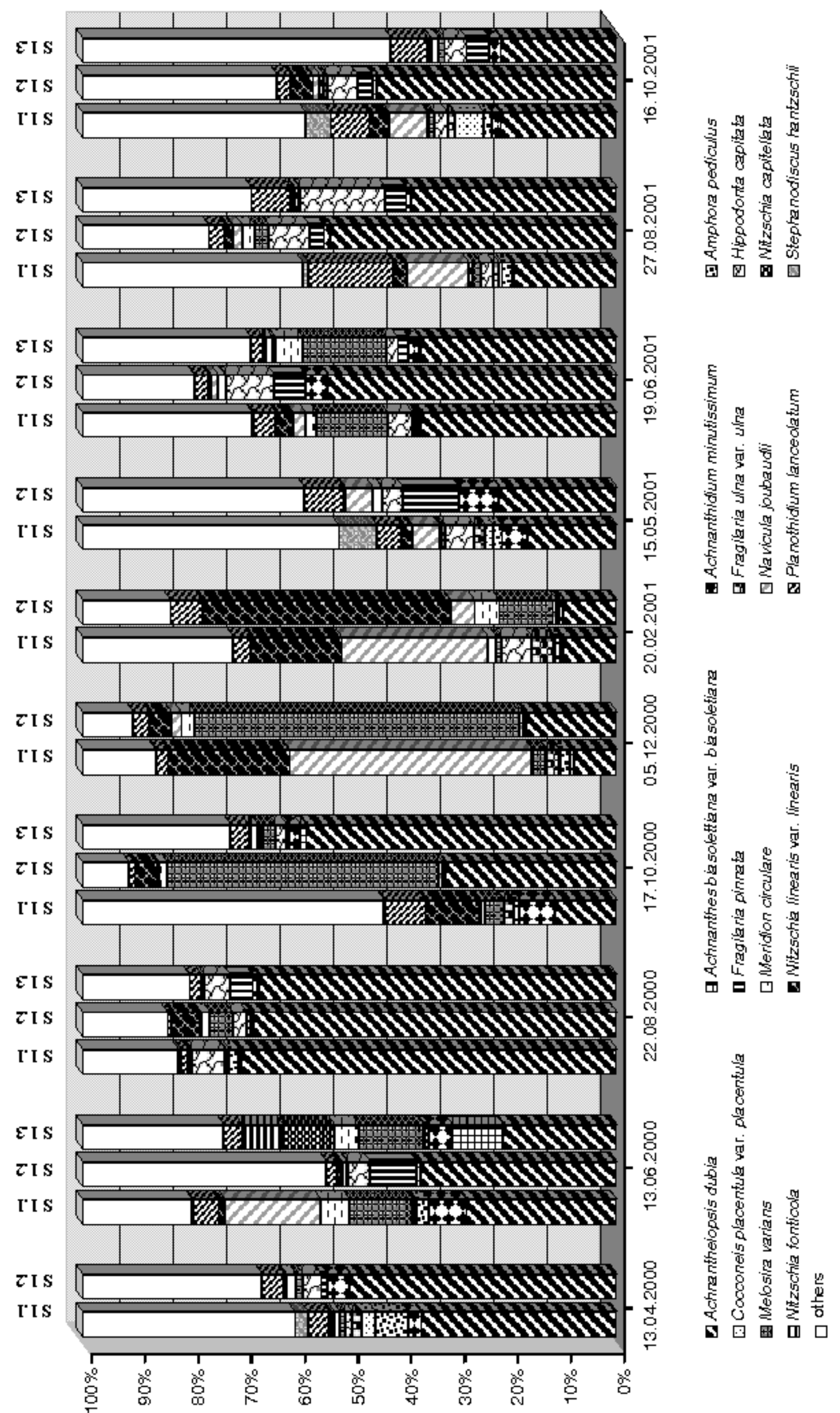


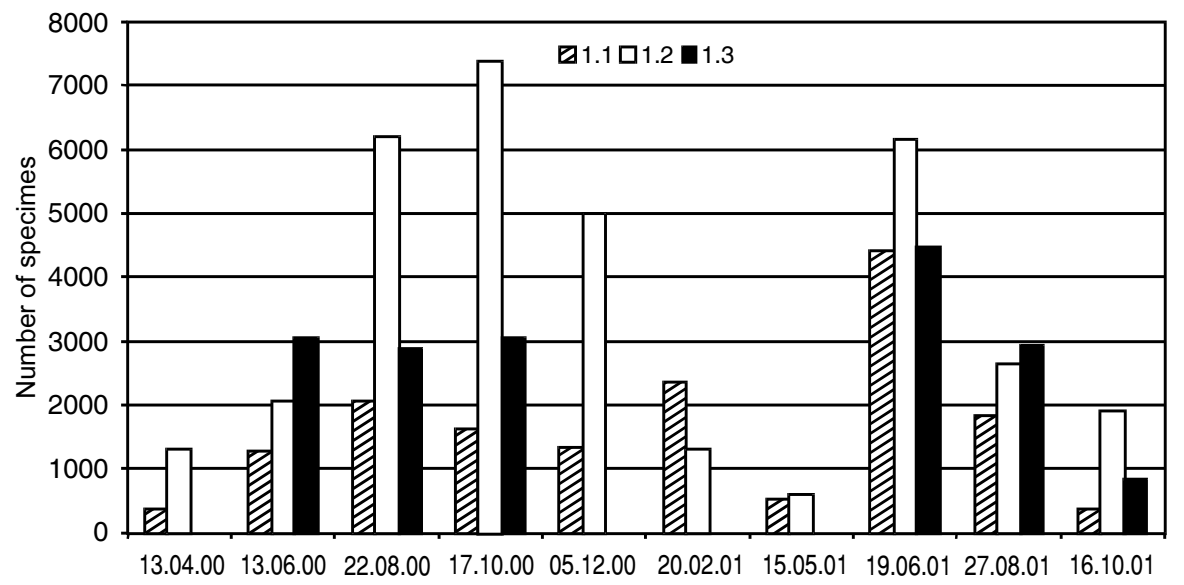

Fig. 7. Number of specimens in individual samples.

Macroalgae

Eleven species of macroalgae belonging to the phyla Chlorophyta, Chrysophyta, Rhodophyta were determined in all the samples.

The phylum Chlorophyta is represented by four taxa: Draparnaldia acuta (Agarth) Kütz, Stigeoclonium subsecundum Kütz., Ulothrix sp. Kütz., Vaucheria sessilis (Vaucher) De Candolle. Three species were identified in the phylum Chrysophyta: Tribonema elegans Pascher, Tribonema viride Pascher, Tribonema vulgare Pascher, The phylum Rhodophyta is represented by three species: Batrachospermum moniliforme Roth, Batrachospermum vagum (Roth) Ag., Chantransia chalybaea (Roth) Fries.

In site 1.1, 7 species of macroalgae were determined. Two species occurred exclusively in the outflow. These are: $B a$ trachospermum moniliforme, Chantransia chalybaea. Eight species occurred in site 1.2, 3 of them were found exclusive-
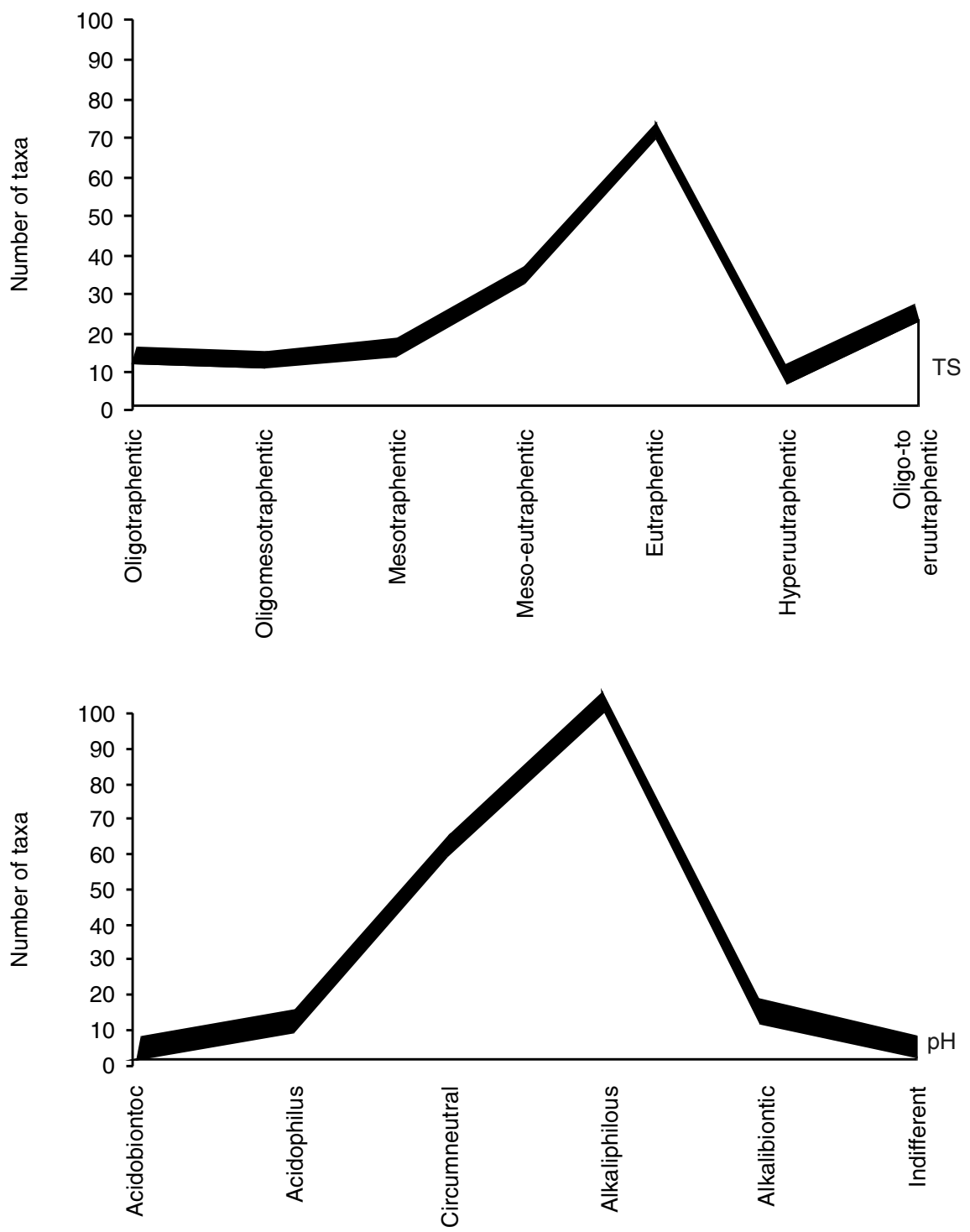

Fig. 8. Ecological indicator value of diatoms in the springs niche. 
ly one meter from the outflow. These are: Batrachospermum vagum, Tribonema elegans, Tribonema viride. In site 1.3, 4 species of macroalgae were noted: Stigeoclonium subsecundum, Ulothrix sp., Tribonema vulgare, Vaucheria sessilis.

\section{Plant associations}

The spring niche was charted on each occasion and the vegetation distribution was presented on a map in the scale 1:20 (Fig. 9).

The type of the community Ranunculo - Sietum erecto submersi (Roll. 1939) Mull. 1962 was determined on the basis of Berula erecta (Huds.) Coville, Callitriche cophocarpa Sendtner and Elodea canadensis Rich. species. The Ranunculo - Sietum erecto - submersi association occurs in very clean and well oxygenated waters on the limestone substrate; in the upland's areas and in the foothills of the Sudeten and the Carpathian Mountains, as well as in young glacial regions, in streams flowing on the till substrate rich in calcium (Matuszkiewicz 1981).

The Ranunculion fluitantis association comprises ecologically specialised communities of hydrophytes rooted in the bottom of flowing waters. They occur in the lowland and in the mountains: in rivers, streams and brooks with different current flow rates and current force and different water properties, and show a high differentiation dependent on the local habitat factors and a low regional variability. Communities forming the Ranunculion association may be significant bioindicators of water pollution in rivers and thus should be examined more closely from the point of view of phytosociology and ecology. These communities are underexplored in Poland (Matuszkiewicz 1981).

The order Potamogetonetalia represents communities of freshwater macrophytes in meso- and eutrophic inland water reservoirs (Matuszkiewicz 1981).

The observed changes in the distribution of vegetation patches are indicative of the process of replicative secondary succession (Falińska 1996). The Ranunculo - Sietum erecto - submersi association remains at the first succession stage - initial stage. Amplitude of the water level fluctuations is the most fundamental environmental condition that inhibits the development of next succession stages.

The following species of bryophytes were collected around the spring niche: Brachythecium salebrosum (Web. et Mohr) Schimp., Bryum pseudotriquetrum (Hedw.) Gaertn., Meyer et Scherb., Calliergonella cuspidata (Hedw.) Loeske, Leptodictyum riparium (Hedw.) Warnst., Marchantia polymorpha var. aquatica Nees, Plagiomnium ellipticum (Brid.) T. Kop, Pseudoscleropodium purum (Hedw.) Fleisch. in Broth.

\section{DISCUSSION}

The examined spring was characterized by constant physical and chemical conditions throughout the study period. However, the sudden changes brought by the rise of the water level in the spring during the flood in the Warta River valley occurred periodically.

Other authors show that the constancy of conditions is typical for springs, which undoubtedly differentiates them from surface waters (Odum 1971).

Three microhabitats, the outflow, a stone (situated 0.5 $\mathrm{m}$ from the outflow) and one meter from the outflow, were examined in the spring niche. Both the outflow and the site located one meter from it are situated in the spring mainstream, while the stone is located outside the mainstream, on the left bank of the spring niche. The substrate in the outflow consists of fine rock material and sand; sand is the substrate one meter from the outflow.

Cantonati and Pipp (2000) researched Borzago and Malaga Nambi Mountain streams in the Adamello-Brenta Regional Park in the Southern Alps. They established three sampling sites in each case; the first one - spring, the two other some few hundred meters downstream. As they observed, diatoms were characterised by a greater diversity downstream.

The spring outflow is a more stable habitat than those situated downstream. Habitat differentiation is observed along the stream. Downstream sections are characterised by unstable conditions such as floods, fast current, glacial formations, river acceleration and deceleration as well as temperature. These factors influence the algae communities. The author suggests that the faster current in the upstream sections may reduce species diversity and limit the accumulation of periphyton, which leads to an impoverishment of algae species as a result of a strong selection of rheophile species (Cantonati 1999, 2001).

The results of the studies on the spring in Działoszyn show an increase in the diversity of diatom communities in microhabitats as the distance from the spring increases. Fewer taxa occurred in the outflow and the frequency there was lower than that for one meter from the outflow. The number of species and individuals on the stone was greater than that in the outflow, but smaller than that, one meter from the outflow.

The diatom community in the outflow was characterised by a high diversity: 175 determined taxa. Waszkiewicz (1999) determined the total number of 77 species in her study of three karst springs in the Warta valley near Częstochowa. Such a relatively high diversity in the examined spring may be connected with the hydrological type of the spring (slope-channel), and its location near an old riverbed.

Budede 1928, Whitford and Schumacher 1963 (after Cantonati 1999) draw attention to the fact that periphyton communities become stable as a result of constant physical and chemical properties. Some researchers point out that seasonal changes of algae in the initial stream course are only slightly significant, while others prove that seasonality is distinctive when light is considered as a factor that brings about seasonal changes (Cantonati and Pipp 2000).

Seasonal variability of the diatom community, conditioned by the duration of the day and access to light, was clearly visible in the studies on the spring in Działoszyn throughout the period of study. The number of individuals and taxa diminished between October and December, when the day was short, while it began to increase in February as the daytime lengthened, despite constant physical and chemical parameters.

In his study on the ecology of cyanobacteria in mountain springs in the southern Alps, Cantonati et al. (1996) showed that the diversity and seasonal variability increased from the spring to a streamlet situated downstream, which suggests that the water current may also play a significant role.

Similar relationships were observed in the spring studied. Seasonal variability was more conspicuous as the distance from the outflow increased. The increases and decreases in the number of individuals and taxa taking place in the outflow were not as drastic as those in the other microhabitats. 
13.04 .2000

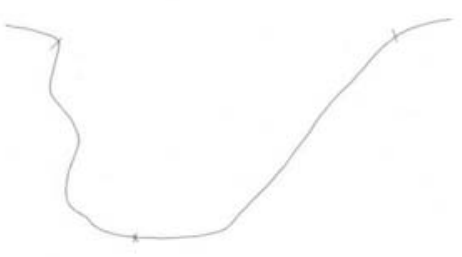

$20020406080100 \mathrm{~cm}$

17.10 .2000

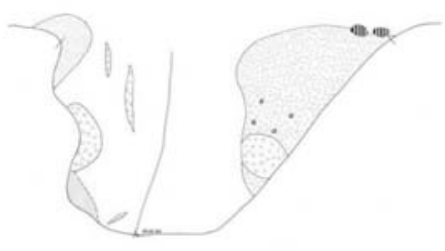

$20020406080100 \mathrm{~cm}$

24.04.2001

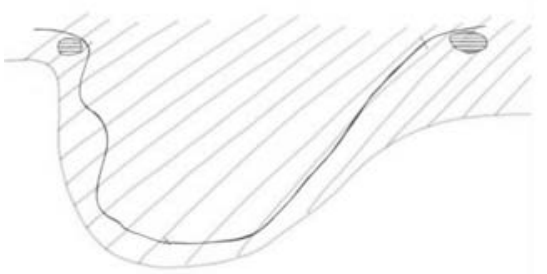

$20020406080100 \mathrm{~cm}$

27.08.2001

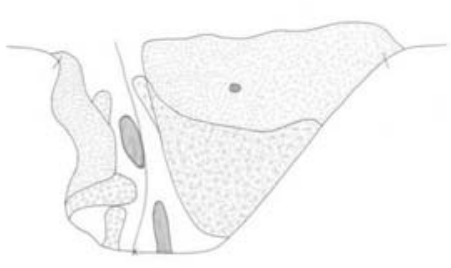

$20020406080100 \mathrm{~cm}$
13.06 .2000

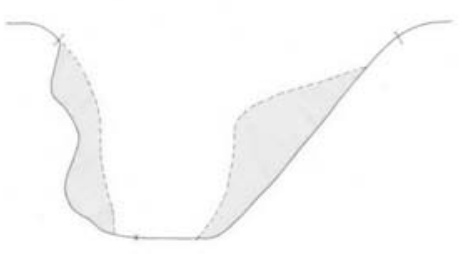

$20020406080100 \mathrm{~cm}$

05.12 .2000

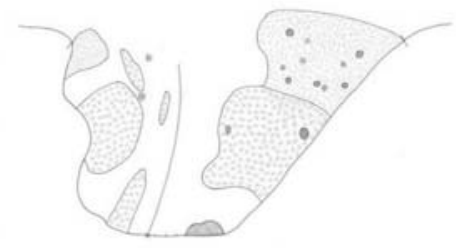

$20020406080100 \mathrm{~cm}$

15.05.2001

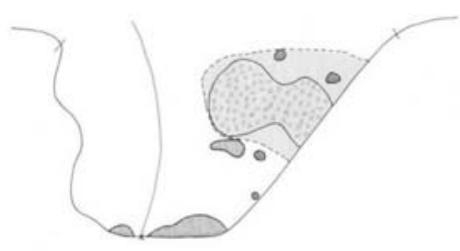

$20020406080100 \mathrm{~cm}$

16.10.2001

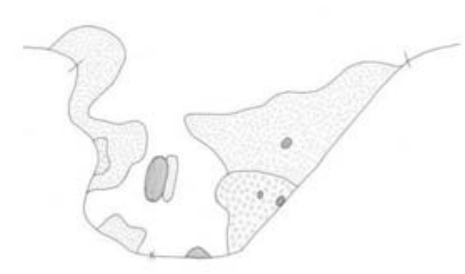

$20020406080100 \mathrm{~cm}$
22.08 .2000

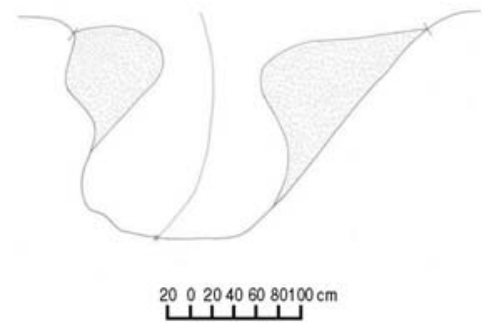

20.02.2001

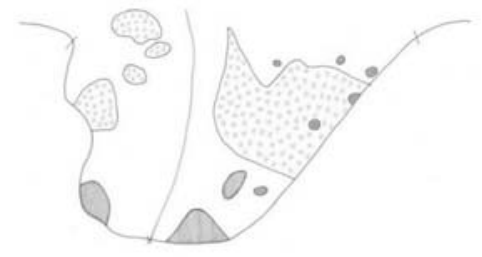

$20020406080100 \mathrm{~cm}$

19.06.2001

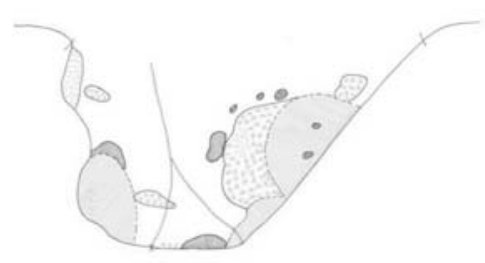

$20020406080100 \mathrm{~cm}$
Scale 1:20

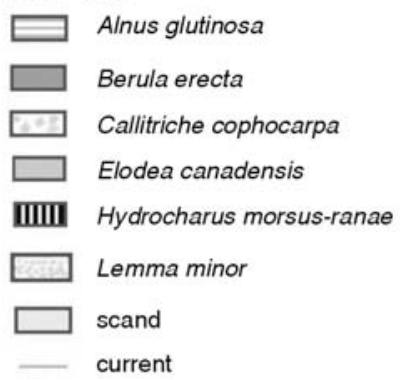

Fig. 9. Distribution of the vegetations flats in the spring niche from April 2000 till October 2001.

The flood in the Warta catchments in April 2001 was a catastrophic event in the representation of diatom communities in the spring studied. The spring niche changed completely after the waters of the Warta River flew into the spring. Consequently, the frequency of diatoms in the outflow and one meter from the outflow was similar in May.

The flood also affected the species composition of macroalgae. A species exchange took place between individual microhabitats. Tribonema vulgare and Ulohtrix sp. occurred in the outflow after the flood. Draparnaldia acuta and Stigeoclonium subsecundum, not recorded there before, occurred one meter from the outflow. Batrachospermum vagum, a species carried in by the water current, not recorded in any of the microhabitats before, occurred. These species disappear in habitats alien to them, and in October 2001 they were absent in the sampling sites in which they had been recorded earlier.

The development of the vegetation cover depends of habitat conditions (Siedlecka-Binder 1967). Even, amplitude of water level fluctuations, not only a flood, is one of the 
major factors conditioning seasonal variability of vascular plants. Vegetation patches undergo qualitative and quantitative changes in particular months. These conditions prevent the development of successive succession stages, as a result of which the Ranunculo - Sietum erecto - submersi association remains at the first succession stage.

Researchers of springs have attempted to decide whether stable conditions in springs select organisms specialised for the habitat (Round 1960). Some studies on diatom communities in springs show that these communities do not have specific species. In the case of diatoms, however, such species are recorded in springs (Cantonati 1996).

In his comparison of the algoflora of a spring with that of a small pond in a botanical garden, Round (1960) proved that both floras were generally similar. In his studies on other springs in England, he also showed that diatom flora consisted of species characteristic of flowing waters, and there were no algae occurrence would be limited to this habitat.

On the basis of determined domination of species in the spring outflow, such as Achnantheiopsis dubia, Achnanthes biasolettiana, Achnanthidium minutissimum, Amphora pediculus, Cocconeis placentula var. placentula, Fragilaria pinnata, F. ulna var. ulna, Hippodonta capitata, Melosira varians, Meridion circulare, Navicula joubaudii, Nitzschia capitellata, N. fonticola, N. linearis var. linearis, Planothidium lanceolatum, Stephanodiscus hantzschii, it can be concluded that these species are not characteristic for springs, even though they are recorded there; they are also found in other water ecosystems. Ecological indicator values of $\mathrm{pH}$ and trophic state of dominant diatom species reveal alkaline and eutrophic water features.

The occurrence of Navicula joubaudii in springs has been rarely noted (Wojtal 2001; Rakowska 2001) while it is one of the dominant taxa in the studied spring. The species forms communities with Diadesmis perpusilla and Nitzschia amphibia: these species occurred in the outflow and are accessory. Navicula joubaudii is an indicator of oligosaprobic to $\beta$-mesosaprobic waters, with small organic matter content (Krammer and Lange-Bertalot 1986). Wojtal (2001) noted Navicula joubaudii on mud of a karstic spring and among filamentous thalli of Cladophora sp. in the polluted part of the stream, where conductivity was medium 360-480 $\mu \mathrm{S}$, and pH 6.1-8.0. Rakowska (2001) noted this species in two river springs (Bzura and Rawka) and in the limnokrenic karstic spring "Niebieskie Źródła". The species preferences were determined as oligotrophic in respect trophy, and alkaliphilic in respect of $\mathrm{pH}$ value. In the spring studied Navicula joubaudii was dominant and constant species; its presence can indicate alkaliphilic and oligo- to eutrophic water conditions.

Other authors suggest, however, that springs represent a geographically isolated system that can be a potential place of preservation of relic or endemic species. Such studies were conducted in springs in northern America: Florida, Utah, Texas, and Mexico, where the occurrence of endemic species was recorded (Alison and Sheath 1999).

Cantonati and Ortler (1998) shows in his studies that communities of algae in springs are characterised by these species that are often found in other springs and in similar habitats; however they are not, crenobionts or species limited to clean fast flowing waters. Cantonati and Pipp (2000) suggest that communities of algae should be considered in terms of the presence of crenophyles or as species known little that are usually subdominants, and are mostly in the group of rare species.

In the study on the spring in Działoszyn, the similarity between these three communities was determined to be high based on the qualitative comparative index of diatom communities, the Kulczyński coefficient, and ranges between 0.71 to 0.78 .

Cantonati (2001) suggests that preferences of individual species are more important, if there are no obvious differences between diatom communities. Similar suggestions appear in Rakowska's (1996) article: in order to determine the conditions occurring in a habitat, the significance of species with a narrow tolerance range is greater than those that develop abundantly and whose tolerance for physical and chemical conditions in water is broad.

The analysis of rare species with a narrow tolerance range, conducted in this study, determines the habitat type. Achnanthes biasolettiana var. subatomus, A. biasolettiana var. biasolettiana, A. lauenburgiana, A. rossi, Amphora fogediana, Eunotia incisa var. incisa, Gomphonema angustum, G. tenue, Diadesmis perpusilla, Fallacia lenzii, Nitzschia acidoclinata, Stauroneis lauenburgiana, were rare species (below $1 \%$ of individuals) in the outflow. These species represent low constancy classes, chiefly I and II, and sporadically class III. Consequently, based on these species the place of the outflow can be determined as an oligo- to mesotrophic habitat, with low organic matter content, well oxygenated water, high limestone content, medium and high electrolyte presence, low sulphate content, $\mathrm{pH}$ neutral to alkaline.

Dominant species, which have clear $\mathrm{pH}$ and trophic preferences, can be used as indicators of alkaliphilous (4) and eutraphentic (5) features of water. We suggest that species co-occurring constantly in the studied spring (V class): Achnantheiopsis dubia, Amphora libyca, Navicula exilis, Navicula moskali, Navicula upsalensis, which do not have assigned indicator values, can also be treated as indicators of these water conditions.

\section{ACKNOWLEDGMENTS}

This study was supported by the State Committee for Scientific Research, grant no. 3P04G 05732. The authors' are very grateful to Prof. L. Kucharski for his critical consultation of this manuscript.

\section{LITERATURE CITED}

ALISON R., SHEATH S., SHEATH R. 1999. Seasonality of macroalgae and epilithic diatoms in spring - fed streams in Texas, USA. Hydrobiol. 390: 73-82.

CANTONATI M. 1999. Distribution and seasonality of the phytobentos along two mountain spring streams in catchments of contrasting lithology. Suppl. Boll. Mus. Civ. Stor. Nat. Venezia 43: 357-362.

CANTONATI M. 2001. The diatom communites of the liverwort Chiloscyphus polyanthos var. rivularis in a mountain spring fed stream in the Adamello - Brenta Regional Park (Northern Italy) In: R. Jahn, J.P. Kociolek, A. Witkowski and P. Compčre (eds). Lange-Bertalot-Festschrift: Studies on Diatoms. A.R.G. Gantner: 353-368.

Cantonati M., Ortler K. 1998. Using spring biota of pristine mountain areas for long-term monitoring. Hydrology, Water Resources and Ecology in Headwaters. Merano: 379-385. 
CANTONATI M., PIPP E. 2000. Longitundial and seasonal differentiation of epilithic diatom communities in the uppermost sections of two mountain spring- fed streams. Verh. Internat. Verein. Limnol. 27: 1591-1595.

CANTONATI M., ROTT E., PIPP E. 1996. Ecology of cyanophytes in mountain springs of the Rivera Sarca catchment (Adamello - Brenta Regional Park, Trentino, Northern Italy). Algolog. Studies 83: 145-162.

DYNOWSKA I. 1983. Springs within the Upland of CracowWieluń and Miechów. Stud. Document. Centre Physiogr. Pol. Acad. Sci. 11: 1-130.

FALIŃSKA K. 1996. Ekologia roślin - podstawy teoretyczne, populacja, zbiorowisko, procesy. PWN, Warszawa: 1-453. (in Polish)

Kawecka B., Eloranta P.V. 1994. Zarys ekologii glonów wód słodkich i środowisk lądowych. PWN, Warszawa: 1-252. (in Polish)

KLECZKOWSKI A. 1972. Wody powierzchniowe i podziemne Wyżyny Krakowsko-Wieluńskiej. In: J. Litwiniszyn (ed.) Studia ośrodka dokumentacji fizjograficznej. PWN, Warszawa: 31-68. (in Polish)

KRAMMER K., LANGE-BERTALOT H. 1986. Bacillariophyceae 1. Naviculaceae. In: H. Ettl, J. Gerloff, H. Heyning, D. Mollenhauer (eds), Süsswaserflora von Mitteleuropa. Veb G. Fischer Verlag, Stuttgart-New York: 1-876.

KRAMMER K., LANGE-BERTALOT H. 1988. Bacillariophyceae 2. Bacillariaceae, Epithemiaceae, Surirellaceae. In: H. Ettl, J. Gerloff, H. Heyning, D. Mollenhauer (eds) Süsswaserflora von Mitteleuropa.Veb G. Fischer Verlag, Jena: 1-611.

KRAMMER K., LANGE-BERTALOT H. 1991a. Bacillariophyceae 3. Centrales, Fragilariaceae, Eunotiaceae. In: H. Ettl, J. Gerloff, H. Heynnig, D. Mollenhauer (eds) Süsswaserflora von Mitteleuropa. Veb G. Fischer Verlag, Jena: 1-600.

KRAMMER K., LANGE-BERTALOT H. 1991b. Bacillariophyceae 4. Achnanthaceae, Kritische Ergänzungen zu Navicula (Lineolatae) und Gomphonema Gesamtliteraturverzeechnis Teil 1-4. In: H. Ettl., J. Gerloff, H. Heyning, D. Mollenhauer (eds), Süsswaserflora von Mitteleuropa. Veb G. Fischer Verlag, Stuttgart-Jena: 1-437.

KUBIK B. 1970. Okrzemki trzech źródeł potoku Będkówka (Wyżyna Krakowsko-Częstochowska). Fragm. Flor. Geobot. 16 (4): 549-561. (in Polish with English summary)

KUCHARSKI L., FILIPIAK E. 1999. Szata roślinna obszarów źródliskowych środkowej Polski i jej ochrona. In: E. Biesiadka, S. Czachorowski (eds), Źródła Polski, Stan badań, Monitoring i Ochrona. Wyższa Szkoła Pedagogiczna, Olsztyn: 87-94. (in Polish with English summary)

LANGE-BERTALOT H., METZELTIN D. 1996. Indicators of oligotrophy, 800 Taxa representive of three ecologically district lake types. Koeltz Scientific Books, Königstein, Germany: 1-390.

LANGE-BERTALOT H. 2001. Navicula sensu stricte, 10 Genera Separated from Navicula sensu lato, Frustulia. In: H. LangeBertalot (ed.), Diatoms of Europe. A.R.G. Ganther Verlag K.G.: 1-526.

MATUSZKIEWICZ W. 1981. Przewodnik do oznaczania zbiorowisk roślinnych Polski. PWN, Warszawa: 1-298. (in Polish)
ODUM E.P. 1983. Podstawy ekologii. PWRiL, Warszawa: 1-564. (in Polish)

OLACZEK R., CZYŻEWSKA K. 1986. Załęczański Park Krajobrazowy - charakterystyka wartości, ich ochrona i wykorzystanie. Acta Univ. Lodz. Folia Sozol. 2: 1-201. (in Polish with English summary)

RAKOWSKA B. 1996. Diatom communites occurring in Niebieskie źródła near Tomaszów Mazowiecki, Central Poland (1963-1990). Fragm. Flor. Geobot. 41 (2): 639-655.

RAKOWSKA B. 2001. Studium różnorodności okrzemek ekosystemów wodnych Polski niżowej. (Study of diatom diversity in water ecosystems of Polish Lowlands). Wydawnictwo UŁ: 1-77. (in Polish with English summary)

ROUND F.E. 1960. A note on the diatom flora of some springs in the Malham Tarn area of Yorkshire, Arch. Protistenk. 104: 515-526.

SIEDLECKA-BIDNER Z. 1967. Roślinność wodna w potokach Ojcowskiego Parku Narodowego. PAN, Zakład Ochrony Przyrody, Ochrona Przyrody 32: 171-206. (in Polish)

SKALNA E. 1969. Okrzemki trzech źródeł potoku Kobylanka (Wyżyna Krakowsko-Częstochowska). Fragm. Flor. Geobot. 15 (2): 245-254. (in Polish with English summary)

SKALNA E. 1973. Glony wywierzyska krasowego w Jerzmanowicach (Wyżyna Krakowsko-Częstochowska. Fragm. Flor. Geobot. 19 (3): 343-348. (in Polish with English summary)

SKALSKA T. 1966a. Zimowe okrzemki żródła w Dubiu koło Krakowa. Fragm. Flor. Geobot. 12/2: 233-240. (in Polish with English summary)

SKALSKA T. 1966b. Występowanie okrzemek w źródle w Dubiu. Acta Hydrobiol. 8, suppl. 1: 311-319. (in Polish with English summary)

STARMACH K. 1968. Chrysophyta III, Xanthophyceae. In: Flora słodkowodna Polski 7, PAN, Inst. Bot., PWN, Warszawa-Kraków: 1-394. (in Polish)

STARMACH K. 1972. Chlorophyta III: Ulothrichales, Ulvales, Prasiolales, Sphaeropleales, Cladophorales, Chaetophorales, Trentepohliales, Siphonales, Dichotomosiphonales. In: Flora słodkowodna Polski 10, PAN, Inst. Bot., PWN, WarszawaKraków: 1-750.

STARMACH K. 1977. Phaeophyta Rhodophyta. In: Flora słodkowodna Polski 14, PAN, Inst. Bot., PWN, Warszawa-Kraków: 1-445. (in Polish)

SZAFER W., KULCZYŃSKI S., PAWŁOWSKI B. 1986. Rośliny Polski. PWN, Warszawa, 1, 2: 1-1019. (in Polish)

TROJAN P. 1975. Ekologia ogólna. PWN, Warszawa: 1-418. (in Polish)

VAN DAM H., MERTENS A., SINKELDAM J. 1994. A coded checklist and ecological indicator values of freshwater diatoms from the Nertherlands. Nether. J. Aquatic Ecol. 28 (1): 117-133.

WASZKIEWICZ D. 1999. Okrzemki źródeł krasowych przełomu Warty na Wyżynie Częstochowskiej. In: E. Biesiadka, S. Czachorowski (eds), Źródła Polski, Stan badań, Monitoring i Ochrona. Wyższa Szkoła Pedagogiczna, Olsztyn: 227-240. (in Polish with English summary)

WOJTAL A. 2001. New or rare species of the genus Navicula (Bacillariophyceae) in the diatom flora of Poland. Pol. Bot. J. 46 (2): 161-167. 